\title{
The first woman born by in vitro fertilization in Korea gave birth to a healthy baby through natural pregnancy
}

\author{
Sung Woo Kim, MD ${ }^{1,2}$, Minhee Lee, MD ${ }^{1}$, Hoon Kim, MD, PhD ${ }^{1,2,3}$, Seung-Yup Ku, MD, PhD $D^{1,2,3}$ \\ ${ }^{1}$ Department of Obstetrics and Gynecology, Seoul National University Hospital, ${ }^{2}$ Department of Obstetrics and Gynecology, Seoul National University \\ College of Medicine, ${ }^{3}$ Institute of Reproductive Medicine and Population, Medical Research Center, Seoul National University, Seoul, \\ Korea
}

Steptoe and Edwards succeeded in implanting an embryo in Lesley Brown's uterus after fertilizing sperms and oocytes in vitro in November 1977 [1]. Lesley Brown had no fallopian tubes on either side. On July 25, 1978, the world's first in vitro fertilization (IVF) baby, Louis Brown, was born via a cesarean section at Oldham General Hospital in Manchester, UK [1]. It was the moment of hope for couples around the world who were suffering from infertility. Dr. Edwards was awarded the 2010 Nobel Prize in Physiology/Medicine for his contribution to human IVF therapy.

Following the birth of Louise Brown in 1978, live births from IVF were reported in Australia in 1980, the USA in 1981, and France in 1982 [2]. In Korea, the first IVF baby was born in 1985, delivered by Dr. Shin Yong Moon and Yoon Seok Chang et al. at Seoul National University Hospital (Fig. 1) [3]. She was the fourth IVF baby in Asia after those born in Singapore, Japan, and Taiwan and gave great hope to 50,000 domestic infertile couples.

A couple had been married for 4 years, and the fallopian tubes were obstructed after surgery for ectopic pregnancy 2 years prior. The couple succeeded in conceiving through IVF after retrieving oocytes by laparotomy on February 11, 1985. On October 12, 1985, at 5:10 a.m., a set of female and male twins were born through a cesarean section in the delivery room of Seoul National University Hospital. The twins' first cries were heard within 5 minutes of each other. The girl was born first and weighed $2.63 \mathrm{~kg}$. The boy weighed $2.56 \mathrm{~kg}$.

After the birth of the first IVF baby, technological advances continue in the field of reproductive medicine, such as cryopreservation of embryos and oocytes, preimplantation genetic diagnosis, and intracytoplasmic sperm injection. Owing to these technological advancements, the IVF pregnancy rate has improved to as high as $30-40 \%$. In Korea, as of 2015, 47,886 IVF procedures were performed according to government reimbursement program data, and 19,736 were born through IVF in 2016 [4].

The procedures have since been simplified, and the oocyte retrieval efficiency has improved by retrieving oocytes under vaginal ultrasound guidance instead of by laparotomy or laparoscopy. The incidence of IVF complications, such as multiple fetal pregnancies and ovarian hyperstimulation syndrome, has significantly decreased due to the development of top-grade embryo selection technology, single embryo transfer, and advances in controlled ovarian stimulation protocols.

As the success rate of IVF increased and its safety proven, an increasing number of couples have become interested in the fertility of the next generation who had been born through IVF. Louise Brown, the first IVF baby, succeeded in natural pregnancy and gave birth to a healthy baby boy in 2006. This has alleviated the concerns regarding whether women born from IVF have a natural pregnancy.

Recently, the sister of the first IVF twin born in Korea gave birth to a healthy baby in February 2019, through a natural

Received: 2021.01.03. Revised: 2021.04.19. Accepted: 2021.05.02. Corresponding author: Seung-Yup Ku, MD, PhD

Department of Obstetrics and Gynecology, Seoul National University Hospital, 101 Daehak-ro, Jongno-gu, Seoul 03080, Korea

E-mail: jyhsyk@snu.ac.kr

https://orcid.org/0000-0002-6423-854X

Articles published in Obstet Gynecol Sci are open-access, distributed under the terms of the Creative Commons Attribution Non-Commercial License (http://creativecommons. org/licenses/by-nc/3.0/) which permits unrestricted non-commercial use, distribution, and reproduction in any medium, provided the original work is properly cited.

Copyright (C) 2021 Korean Society of Obstetrics and Gynecology 


\section{Obstetrics \& Gynecology Science}

Sung Woo Kim, et al. The first Korean IVF baby's pregnancy

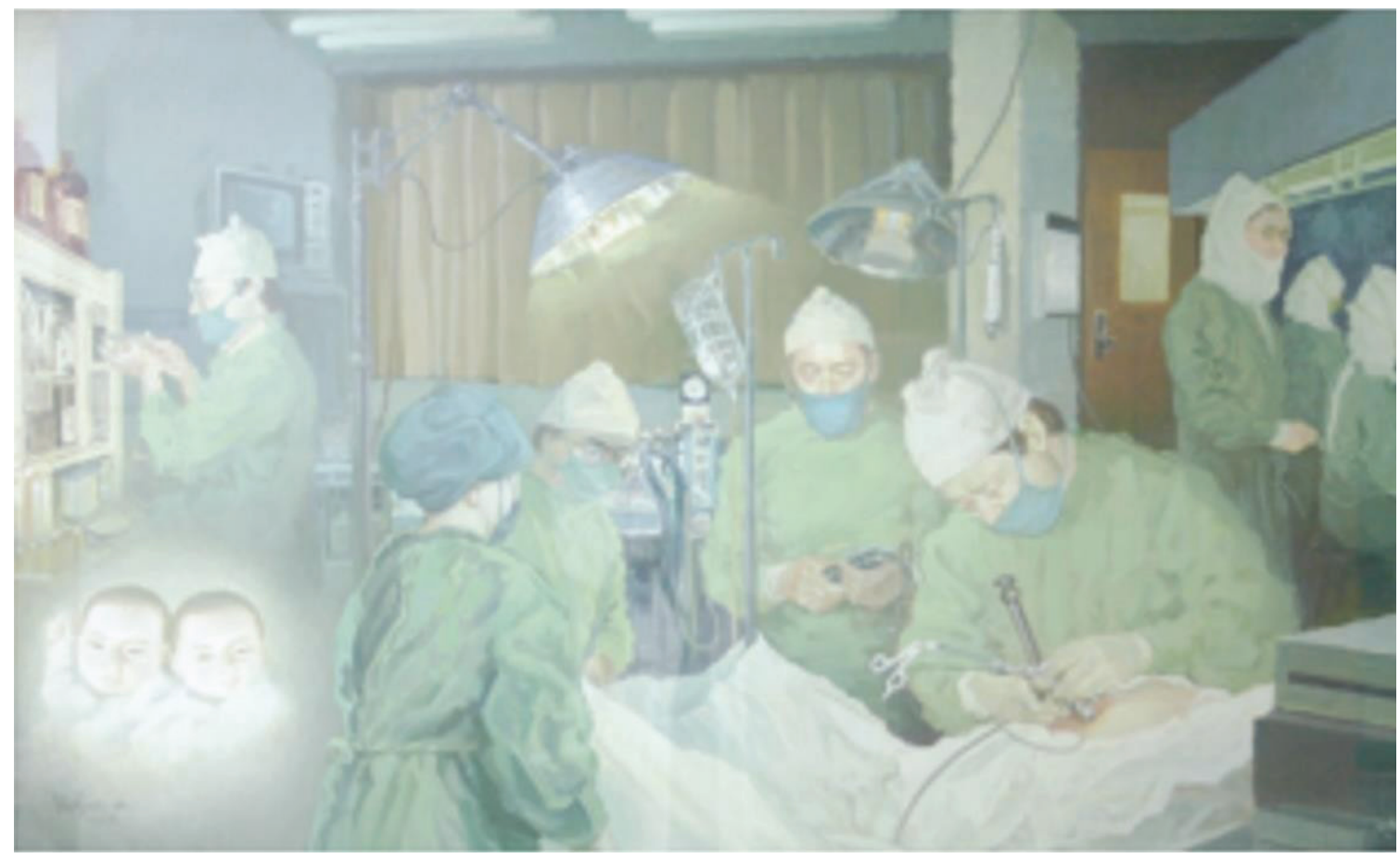

Fig. 1. Painting to commemorate the live birth born through the first in vitro fertilization-embryo transfer in the Republic of Korea (donated by the alumni association of obstetrics and gynecology to Seoul National University Hospital).

pregnancy. She has been having regular menstrual cycles since commencing menarche at 13 years of age. She grew up without any gynecological problems. After a spontaneous pregnancy, regular prenatal check-ups at Seoul National University Hospital did not show any abnormal findings. She visited the delivery room at 39 weeks 2 days of pregnancy with regular labor pain at 5-minute intervals. After expectancy, a $3.165 \mathrm{~kg}$ baby girl was delivered as a normal full-term spontaneous delivery on February 25, 2019, at 2:47 p.m. The baby cried well after birth and showed normal growth without any perinatal problems. This has convinced the public that the generation born through IVF is healthy in terms of fertility, even if they are born through ART procedures.

The first generation of children born through IVF are now adults of reproductive ages. The next generation of babies conceived by these adults is now being born. IVF is no longer an inapproachable, special, and complex procedure. Rather, it is now an easily accessible and common procedure for infertile couples. A woman from Korea's first IVF procedure gave birth to a healthy baby through natural pregnancy and has become an ordinary mother. We will now be able to explore more freely into a new, unknown world based on the accumulated research results and experiences from IVF procedures. Pioneer scientists are currently making innovative attempts, including the reactivation of folliculogenesis through ovarian cortex stimulation, oocyte mitochondrial replacement, in vitro follicle culture, differentiation of oocytes from stem cells, gene editing of gametes and embryos, and ovarian rejuvenation through regenerative medicine [5]. There is no doubt that studies conducted by these passionate explorers will bring more tangible progress in the near future.

\section{Conflict of interest}

No potential conflict of interest relevant to this article was reported.

\section{Ethical approval}

This study does not require approval of the Institutional Review Board because no patient data is contained in this article. The study was performed in accordance with the principles of the Declaration of Helsinki. 


\section{Obstetrics \& Gynecology Science}

Vol. 64, No. 4, 2021

\section{Patient consent}

Written informed consent and the use of images from patients are not required for the publication.

\section{Funding information}

None.

\section{Acknowledgments}

The authors deeply appreciate Professors Yoon Seok Chang, Jin Yong Lee, Shin Yong Moon, Jung Gu Kim, Chang Jae Shin, Young Min Choi, Seok Hyun Kim, and Chang Suk Suh for their pioneering contribution to the field of infertility treatment.

\section{References}

1. Steptoe PC, Edwards RG. Birth after the reimplantation of a human embryo. Lancet 1978;2:366.

2. Cohen J, Trounson A, Dawson K, Jones H, Hazekamp J, Nygren $K G$, et al. The early days of IVF outside the UK. Hum Reprod Update 2005;11:439-59.

3. Chang YS, Lee JY, Moon SY, Kim JK. Pregnancy and its outcome by in vitro fertilization of human oocytes and embryo transfer: a report of the first test tube babe in Korea. Korean J Obstet Gynecol 1986;29:345-61.

4. Ministry of Health and Welfare. Smartcare Infertility-ART in Medical Korea [Internet]. Osong (KR): Korea Health Industry Development Institute; c2019 [cited 2019 Jul 26]. Available from: http://english.visitmedicalkorea.com/ upload/specialize/11/ART.pdf.

5. Annual Capri Workshop Group. IVF, from the past to the future: the inheritance of the Capri Workshop Group. Hum Reprod Open 2020;2020:hoaa040. 